\title{
A ATUAÇÃO DAS ASSISTENTES SOCIAIS VISTA PELAS FAMÍLIAS DAS CRIANÇAS ATENDIDAS EM UM CENTRO PEDIÁTRICO DO CÂNCER
}

\author{
THE ROLE OF SOCIAL WORKERS AS PERCEIVED BY THE FAMILIES OF \\ CHILDREN TREATED AT THE CANCER PEDIATRIC CENTER
}

\author{
Elayne Costa dos Santos ${ }^{1}$ \\ Ana Paula Silveira de Morais Vasconcelos ${ }^{2}$
}

\begin{abstract}
RESUMO
Este artigo apresenta um estudo realizado em um Centro Pediátrico do Câncer, instituição que trata o câncer infanto-juvenil e que é referência estadual. Seu objetivo foi compreender a percepção da atuação das assistentes sociais a partir da visão das famílias das crianças que são atendidas na instituição. Com isso, foi discutida a Lei 8.080 de 1990, que constitui o Sistema Único de Saúde - SUS. Tratou-se da saúde infanto-juvenil, problematizando a Oncologia Pediátrica e suas particularidades, bem como a sua atuação em parceria com uma Associação Filantrópica - entidade sem fins lucrativos - no tratar dessa doença. O artigo também discute a atuação do/a assistente social na saúde. Assim, aponta a importância da ação do profissional e os eixos de atuação do Serviço Social nesta área. Os resultados da pesquisa mostraram que a atuação das assistentes sociais nesse Centro é visível para as famílias das crianças que são atendidas na instituição, porém, em uma concepção de "ajuda” e não na perspectiva da garantia de direitos desses usuários no SUS.
\end{abstract}

PALAVRAS-CHAVE: Sistema Único de Saúde. Oncologia. Oncologia Pediátrica. Serviço Social.

\begin{abstract}
This paper presents a study conducted on a Pediatric Cancer Center (PCC) that is an institution that treats child cancer and that is reference on the state of Ceará. The main goal of this paper is to understand how the patients' families perceive the social workers that work at the institution. With that in mind, we discuss law 8.080 from 1990, that established SUS (the Brazilian Health Care System) and we examine child health care by studying pediatric oncology and the role of PCC in partnership with a nonprofit organization on the treatment of this disease. This paper also discusses the role of the social worker on health care. In that sense, it

\footnotetext{
${ }^{1}$ Assistente social; Pós-graduanda em Especialização em Serviço Social, Políticas Sociais e Seguridade Social. Instituição de origem: Universidade Estadual do Ceará. Telefone: 85 988764452. E-mail: elaynecosta@ymail.com.

${ }^{2}$ Docente do curso de Serviço Social da Faculdade Cearense e Tutora de Núcleo da Residência Integrada em Saúde/Ênfase Saúde da Família e Comunidade da Escola de Saúde Pública do Ceará. Assistente Social, Especialista em Caráter de Residência em Saúde da Família e também especialista Educação na Saúde para Preceptores do SUS. Instituição de origem: Escola de Saúde Pública do Ceará e Faculdade Cearense. E-mail: anapaulasilveira@yahoo.com.br.
} 
shows the importance of this professional and the areas of work of social service on health care. The results of the research showed that the work developed by social workers at CPC is visible to the children's families. However, the families saw that work more as a "help" rather than someone guaranteeing their rights. The social workers did not make it clear that their job is to inform the users of their rights.

KEYWORDS: SUS. Oncology. Pediatric Oncology. Social Work.

\section{INTRODUÇÃO}

O presente artigo apresenta os resultados de um estudo realizado sobre a compreensão da atuação dos profissionais do Serviço Social em um Centro Pediátrico do Câncer, tendo como sujeitos as crianças atendidas, suas famílias e as assistentes sociais. A referida pesquisa foi submetida ao Comitê de Ética e Pesquisa e autorizada através do Parecer n ${ }^{\circ} 693.222$ de 21 de junho de 2014.

A instituição conta com um serviço que funciona no prédio construído por uma Associação Filantrópica. As crianças chegam ao Centro Pediátrico do Câncer através de encaminhamentos de hospitais dos municípios circunvizinhos e de outros Estados. O tratamento contra o câncer dura em torno de 2 anos e meio a 3 anos. Mesmo depois de diagnosticada a cura, a criança permanece em acompanhamento ambulatorial respeitando uma agenda administrada pelo oncologista até os 18 anos de idade.

Em relação à Associação Filantrópica, a mesma surgiu em 1996, quando um grupo de pessoas começou a fazer visitas em um hospital infantil da cidade levando às crianças e adolescentes com câncer um pouco de alegria, utilizando atividades com propostas lúdicas para entretenimento, brincadeiras, jogos recreativos e doações. Com essa dedicação, em 07 de abril de 1997 a direção do hospital infantil fundou, em um anexo do mesmo, um projeto com o objetivo de "transformar a história do câncer infantil”.

De acordo com o Instituto Nacional do Câncer - INCA (2013), o câncer, considerado o mal do século XXI, é a doença que mais mata no mundo. Segundo dados da Organização Mundial da Saúde (2013), é responsável por cerca de 8 milhões de óbitos por ano - e também a principal causa de morte por doença entre crianças e adolescentes brasileiros. Apesar de dados tão sombrios, o diagnóstico de câncer não representa uma sentença de morte, como prova o alto índice de cura do câncer infantil no país: 80\%, segundo dados do Instituto Nacional do Câncer (INCA, 2013). 
Essa pesquisa tem como temática o Sistema Único de Saúde no Brasil e a atenção à criança com câncer; envolve uma discussão em relação à alta complexidade na Rede de Atenção Oncológica, dando ênfase à oncologia infanto-juvenil. É problematizada a atuação da/o assistente social na saúde e os eixos de atuação desses profissionais nessa área.

Contudo, o objetivo primordial do artigo foi compreender a percepção da atuação dos profissionais do Serviço Social no Centro Pediátrico do Câncer, a partir da visão das famílias das crianças que são atendidas na Instituição.

Portanto trata-se de uma pesquisa de natureza qualitativa, na qual o instrumento de coleta utilizado foi um roteiro de entrevista semiestruturada para as profissionais do Serviço Social e outro roteiro para as mães das crianças assistidas pela instituição; utilizou-se a revisão da literatura, indispensável para a pesquisa, que é uma parte vital do processo de investigação, e amostragem foi definida por saturação. As entrevistas foram realizadas na sala do Serviço Social, com as assistentes sociais, e, em um espaço apropriado da instituição com as mães.

\section{O SISTEMA ÚNICO DE SAÚDE (SUS)}

O Sistema Único de Saúde (SUS) se caracteriza como o modelo público de serviços e ações de saúde no Brasil. Levado por um conjunto de princípios e diretrizes para todo o território nacional, inserindo, em sua estrutura institucional, espaços e instrumentos para a democratização e compartilhamento da gestão do sistema de saúde. Sua implantação tem início na década de 1990, em consequência da promulgação da Lei Orgânica da Saúde, lei ${ }^{\circ}$ 8.080, de 19 de setembro de 1990, que foi complementada pela lei no 8.142, de 28 de dezembro de 1990 (BRASIL, 1990).

Para materializar a política de saúde, a Constituição instituiu o Sistema Único de Saúde (SUS) como “o conjunto de ações e serviços públicos de saúde, prestados por órgãos e instituições públicas federais, estaduais e municipais, da administração direta e indireta e das fundações mantidas pelo Poder Público” (BRASIL, 1990).

Assim, incluem-se nessa definição as atividades dirigidas às pessoas que são voltadas para a promoção de saúde e prevenção, diagnóstico, tratamento e a reabilitação de agravos e doença; serviços ambulatoriais, hospitalar e nas unidades de diagnóstico e terapêutico geridos pelo governo e em outros espaços, como o domiciliar; ações de 
diferentes complexidades e custos; as intervenções ambientais, que incluem as condições sanitárias em ambientes nos quais se vive e trabalha e as instituições públicas voltadas para o controle de qualidade, pesquisa e produção de insumos, medicamentos e equipamentos para a saúde (NORONHA et al., 2012).

Os serviços que compõem o SUS têm direção única e seguem os mesmos princípios organizativos em todo o território nacional, sob a responsabilidade das três esferas autônomas de governo federal, estadual e municipal. Dessa forma, o SUS não é um serviço ou uma instituição, mas sim um Sistema, que significa um conjunto de unidades, de serviços e ações que interagem para um fim comum. Esses elementos integrantes do sistema referem-se, ao mesmo tempo, às atividades de promoção, proteção e recuperação da saúde.

O SUS não é composto somente por serviços públicos, mas por uma rede de serviços que são privados, inclusive hospitais e unidades de diagnose e terapia, que são remunerados com recursos públicos destinados à saúde, entre os quais podemos situar o Centro Pediátrico de tratamento do Câncer.

De acordo com o artigo 198 da Constituição Brasileira de 1988, as ações e serviços públicos de saúde integram uma rede regionalizada e hierarquizada e constituem um sistema único, organizado de acordo com as seguintes diretrizes: descentralização, com direção única em cada esfera de governo; atendimento integral, com prioridade para as atividades preventivas, sem prejuízo dos serviços assistenciais; participação da comunidade, ou seja, haverá direção federal, estadual, municipal e do Distrito Federal, conferindo, assim, o caráter descentralizado; todas as necessidades médicas deverão ser oferecidas pelo serviço público de saúde e a comunidade participa na gestão da saúde (BRASIL, 1988).

Ainda, o artigo 199 da Carta Magna descreve que a assistência à saúde é livre à iniciativa privada, que as instituições privadas poderão participar de forma complementar do Sistema Único de Saúde, segundo diretrizes deste, mediante contrato de direito público ou convênio, tendo preferência as entidades filantrópicas e as sem fins lucrativos. (BRASIL, 1988). É essa prorrogativa que permite a atuação de Associação Filantrópica, instituição sem fins lucrativos que atua no Centro Pediátrico de tratamento do Câncer, com eixo no tratamento do câncer infanto-juvenil.

O artigo constitucional citado acima e a ofensiva neoliberal vivenciada no Brasil constituem um projeto de sociedade adverso ao debatido pelo Movimento de Serv. Soc. \& Saúde, Campinas, SP v. 14, n. 2 (20), p. 261-278, jul./dez. 2015 ISSN 1676-6806 
Reforma Sanitária, esse conjunto de ações promovem a redução das políticas públicas e a transfere para a sociedade civil, ponto a se considerar negativo, pois o Estado diminui a oferta de serviços e, de certa maneira, passa a responsabilidade social para a sociedade.

São princípios do SUS descritos na Lei Orgânica da Saúde: a universalidade de acesso em todos os níveis de assistência; igualdade na assistência à saúde, sem preconceitos ou privilégios de qualquer espécie; integralidade da assistência; participação da comunidade; descentralização político-administrativa (NORONHA et al., 2012).

Esses dispositivos citados têm implicações para a inserção do SUS como política de Estado, tais como: a responsabilidade pela situação de saúde não se torna apenas setorial; as políticas econômicas e sociais devem estar orientadas para eliminar ou reduzir os riscos para a saúde; a integração das políticas de saúde com as demais políticas públicas é de fundamental importância; a atuação integrada das três esferas de governo no âmbito do SUS faz-se também necessária.

Em relação à gestão pública da saúde, esta é exercida por diversos entes governamentais e não-governamentais e requer a valorização e o funcionamento adequado dos espaços dos interesses da sociedade. Assim, podem-se identificar quatro grupos de funções gestoras em diversos campos da atenção à saúde, são eles: formulação de políticas e planejamento; o financiamento; regulação; prestação direta de ações; e serviços de saúde (NORONHA et al., 2012).

Desse modo, com o intuito de concretizar a diretriz do SUS de participação da população, a lei $\mathrm{n}^{\circ}$ 8.142, de 1990, estabelece duas instâncias colegiadas: as Conferências de Saúde e os Conselhos de Saúde compostos com três ideias principais, que são: a necessidade de concretização do controle social, que revela o objetivo do controle da sociedade sobre o Poder Público e também as políticas de saúde; construção de uma gestão participativa, com a implantação da política de saúde pelos gestores e compartilhada com a sociedade; e a necessidade de propiciar melhora na relação entre a sociedade e as instituições públicas na saúde (NORONHA et al., 2012).

Diferentes lógicas de organização dos serviços de saúde podem ser observadas no SUS, segundo vários critérios, tais como os tipos de serviços envolvidos: ambulatoriais, hospitalares de diversos tipos, urgência e emergência, domiciliares; os 
níveis de complexidade: atenção básica, média e alta complexidade; direcionalidade das ações envolvidas: agravos, grupos populacionais e áreas específicas da atenção à saúde.

\section{ATENÇÃO À SAÚDE INFANTO-JUVENIL E ASSISTÊNCIA À CRIANÇA COM CÂNCER}

A saúde da criança e do adolescente, em especial a da criança, tem registrado avanços relevantes no Brasil nas últimas décadas. No ano de 2012, um dos Objetivos do Milênio, relacionado com a mortalidade infantil, foi alcançado antes da época prevista. A meta era de que as 58 mortes registradas para cada mil crianças em 1990 fossem reduzidas para 19 por grupo de mil em 2015. No final de 2011, a taxa já era de 16 para cada mil crianças (BRASIL, 2012 apud CUNHA et al., 2014).

A Organização das Nações Unidas (ONU) define a fase da juventude entre os 15 e 24 anos de idade, mas deixa a possibilidade de que outras diferentes nações definam de outra maneira (EISENSTEIN, 2005 apud CUNHA et. al., 2014). Organização Mundial da Saúde (OMS), adolescente se encontra entre os 10 e 20 anos de idade (WHO 2012 apud CUNHA et al., 2014). O Artigo $2^{\circ}$ do Estatuto da Criança e do Adolescente - ECA - define criança, para os efeitos desta Lei, a pessoa até doze anos de idade incompletos, e adolescente aquela entre doze e dezoito anos de idade (BRASIL, 1990b).

Em 2005, o Ministério da Saúde apresentou a Agenda de Compromissos com a Saúde Integral da Criança e a Redução da Mortalidade Infantil, na qual propôs a criação de uma rede integrada de assistência à criança, organizada em linhas de cuidado e identificando as principais diretrizes de responsabilidade dos governos municipais e estaduais. Assim, fica evidente o desafio do poder público de reduzir as iniquidades, ampliando o acesso a bens e serviços de qualidade, inclusive os de saúde, com a execução de políticas direcionadas pelos princípios da equidade e da universalidade, de modo que alcancem toda a população, beneficiem aqueles que apresentem maior risco de morte e aumentem a chance de sobrevivência na fase da infância (CUNHA et al., 2014).

O desenvolvimento das crianças pode ser seriamente prejudicado se ocorre ativação excessiva ou prolongada do sistema de resposta ao estresse do organismo com potenciais danos para a aprendizagem, o comportamento e a saúde ao longo da vida. Aprender a lidar com as adversidades é um componente essencial do desenvolvimento 
infantil sadio. Os ambientes familiares de apoio podem ajudar as crianças a superar os efeitos indesejáveis da situação estressante por intermédio de respostas saudáveis (WALKER et al, 2011, apud CUNHA, 2014, p. 548).

Segundo Cunha et al. (2014), para propor ações estratégicas, deve-se analisar a prevenção dos agravos e das doenças que ofendem crianças e adolescentes através de dois patamares: o macrossistema e o microssistema. No macrossistema, as ações e estratégias estão ligadas às políticas para o sistema de saúde, que são implementadas pelos governos municipais, estaduais e federal. Essas ações competem, em termos de recurso do orçamento, com outras ações de outros sistemas, como, por exemplo, o sistema educacional.

Assim, as ações de promoção da saúde que estiverem sendo implementadas ao mesmo tempo e integrada pelo sistema educacional, além do sistema de saúde, não somente em relação à saúde da criança e do adolescente, mas com impacto nesses segmentos, com políticas integradas para prevenção de doenças e agravos à saúde têm maior chance de serem efetivas e de obter êxito.

\section{ONCOLOGIA PEDIÁTRICA}

Na área de Oncologia, o Sistema Único de Saúde (SUS) é estruturado para atender de uma forma integral e integrada os pacientes que necessitam de tratamento de neoplasia maligna, este é assegurado, por meio de uma Rede de Atenção Oncológica, cujo planejamento, organização e o controle são de responsabilidade das Secretarias de Saúde estaduais e municipais.

O Ministério da Saúde, por intermédio da Rede de Atenção Oncológica, almeja que a pessoa com câncer tenha um tratamento integral, uma vez que são raros os casos de câncer que precisam de apenas uma modalidade terapêutica oncológica (cirurgia, radioterapia, quimioterapia ou iodoterapia) BRASIL (2010). De acordo com a diretriz da política, o melhor a ser feito é que sejam atendidos em hospitais especificamente credenciados e habilitados que reúnam as condições necessárias de infraestrutura, de recursos humanos e materiais e de equipamentos (BRASIL, 2010).

O INCA (2011) conceitua o câncer como uma doença genética caracterizada pela divisão e proliferação desordenada de células que sofreram mutação em seu material genético. Ele ocorre em qualquer parte do organismo e é o acúmulo das células 
que dão origem aos tumores. Os tumores são caracterizados pelo agrupamento de células anormais, que uma vez formadas serão destruídas pelo organismo, permanecerão como tumores benignos ou transformar-se-ão em tumores malignos. Tudo dependerá do sistema imunológico do indivíduo, que será influenciado por diversos fatores de risco. É a principal doença causadora de morte em crianças com menos de 15 anos de idade (INCA, 2011).

De acordo com o Instituto Nacional de Câncer (INCA, 2013), o câncer atinge 10 em cada 1.000.000 crianças a cada ano, em todo o mundo, sendo que uma criança em cada 600 pode desenvolvê-lo durante a infância, porém hoje se sabe que dois terços desses cânceres são considerados curáveis se o diagnóstico for precoce e o tratamento administrado for adequado ao tipo de câncer.

No Brasil, o câncer já representa a primeira causa de morte por doença entre crianças e adolescentes de 1 a 19 anos, para todas as regiões. Nas últimas quatro décadas, o progresso no tratamento do câncer na infância e na adolescência foi extremamente significativo. Hoje, em torno de $70 \%$ das crianças e adolescentes acometidos de câncer podem ser curados se diagnosticados precocemente e tratados em centros especializados. A maioria deles terá boa qualidade de vida após o tratamento adequado (INCA, 2013).

As faixas etárias pediátricas mais precoces ( 0 a 4 anos) são as mais propensas ao desenvolvimento de câncer, com exceção de linfomas, carcinomas e tumores ósseos, que predominam em crianças entre 10 e 14 anos. As estatísticas da Associação de Apoio à Criança com Câncer (AACC), de 2001 a 2007, mostram que 40\% dos pacientes atendidos está na faixa de 06 a 15 anos e, portanto, em idade escolar (AACC, 2007).

Os sintomas do câncer infantil podem ser facilmente confundidos com outros de doenças comuns na infância, o que pode retardar a procura de um pediatra e, consequentemente, a detecção precoce da doença, tornando-se, assim, fundamental a atenção dos pais a qualquer um dos sintomas e, no caso do surgimento de algum deles, a procura de um especialista para que o mesmo possa fazer o diagnóstico correto (NÚCLEO DE APOIO À CRIANÇA COM CÂNCER, 2013).

O diagnóstico do câncer infantil deve ser feito precocemente, com a finalidade de impedir que a doença se agrave e com isso o prognóstico seja ruim. Ao contrário dos adultos, não existem exames de prevenção que, feitos rotineiramente, detectem a 
manifestação do câncer (CARDOSO, 2007). Daí a importância da avaliação periódica de um pediatra durante toda a infância.

As tecnologias de detecção e tratamento do câncer infantil evoluíram muito nas últimas décadas, com exceção da prevenção, ao contrário dos adultos, já que nestes, a doença está associada a fatores ambientais. Além disso, o diagnóstico precoce muitas vezes não ocorre, pois, em geral, como já foi dito, os sintomas são muito semelhantes aos de outras doenças comuns na infância (INCA, 2013).

As condutas terapêuticas utilizadas no tratamento do câncer costumam ser a cirurgia, a quimioterapia, a radioterapia e o transplante de medula óssea no caso das leucemias (CARDOSO, 2007). Geralmente, estas terapêuticas são utilizadas de forma associada, e a escolha por cada uma delas, bem como a frequência e o tempo de utilização, dependerá de fatores como o tipo de câncer, a localização do tumor, o estágio de evolução da doença, o perfil do paciente, entre outros.

A criança com câncer, mesmo a de menor idade, sente necessidade de saber o que está acontecendo consigo. De algum modo, ela percebe que seus pais estão angustiados e que algo grave e aparentemente sem controle ocorre em seu corpo. A criança sempre acaba sabendo o que tem, mesmo quando a família se esforça no sentido de protegê-la para ocultar-lhe o diagnóstico. O diagnóstico da doença desencadeia reações de choque entre os membros da família. Os pais sofrem intensamente com o descobrimento do câncer na criança ou no adolescente, sendo que a época do diagnóstico é invariavelmente vivenciada pela família, como afirma Valle (1994), "como um tempo de catástrofe, de incertezas, de sentimentos de angústia diante da possibilidade de morte" (ibid., p. 220).

Segundo Lima (1995), desde o momento em que o câncer é comunicado, "profundas alterações nela ocorrem e isto afeta não só seus membros, mas também pessoas do seu círculo de relações" (ibid., p. 89). Para o autor, a criança somente se depara realmente com a doença, no momento em que ela começa a sofrer os efeitos do tratamento, pois ela passa a ter sua vida limitada, não podendo realizar as atividades que anteriormente realizava.

As crianças com câncer e seus familiares têm necessidades especiais que podem ser mais bem atendidas em centros especializados em crianças com câncer. O tratamento do câncer infantil envolve uma equipe multidisciplinar que deve incluir oncologistas pediátricos, cirurgiões pediátricos, radioterapeutas, enfermeiros 
pediátricos, psicólogos, assistentes sociais, nutricionistas e fisioterapeutas, na qual os profissionais estão presentes nas diversas atividades, sem perder suas especificidades. Assim, de acordo com as Abordagens Básicas para o Controle do Câncer - ABC do Câncer - podemos observar:

[...] os tratamentos instituídos devem estar inseridos em uma abordagem multidisciplinar em que outras áreas técnicoassistenciais, como enfermagem, farmácia, serviço social, nutrição, fisioterapia, reabilitação, odontologia, psicologia clínica, psiquiatria e estomaterapia (cuidados de ostomizados), estejam obrigatoriamente envolvidas (INCA, 2011, p. 69)

Visto que todas as etapas de atendimento das crianças são oportunidades de se lidar com os vínculos, com a subjetividade implicada na família e na criança e o desejo e a adesão dos usuários nas atividades oferecidas. Desde o diagnóstico até o fim do tratamento, os profissionais desenvolvem trabalhos de cuidados paliativos que, segundo a Organização Mundial de Saúde, é uma abordagem ou tratamento que melhora a qualidade de vida de pacientes e familiares diante de doenças que ameacem a continuidade da vida (ACADEMIA NACIONAL DE CUIDADOS PALIATIVOS, 2009).

Dentro da perspectiva analisada, identifica-se a importância do trabalho multidisciplinar para as crianças com câncer do Centro Pediátrico de tratamento do Câncer em questão, pois em sua atuação enquanto profissionais que articulados com o projeto elaborado por uma equipe integrada, são perceptíveis os impactos na dimensão da subjetividade dos indivíduos, a partir de uma postura ético-política fortalecendo o processo de superação das situações vivenciadas pelos usuários e pelas famílias. O trabalho em equipe traz novos desafios, exigindo competências e habilidades para o trabalho em grupo e para a justificação clara e objetiva de procedimentos técnicos pertencentes a tal especialidade.

\section{ATUAÇÃO DA/O ASSISTENTE SOCIAL NA SAÚDE}

Os desafios presentes no campo da atuação exigem do (a) profissional o domínio de informações para identificação dos instrumentos a serem acionados e requerem habilidades técnico-operativas que permitam um diálogo com os diferentes segmentos 
sociais. O conhecimento da realidade possibilita a sua decodificação para "iluminar” a condução do trabalho a ser realizado.

Sabemos que o Serviço Social, historicamente, atua nas múltiplas refrações da questão social, conformadas na ordem social contemporânea e seus procedimentos técnicos são instrumentais vinculados a uma intencionalidade, que extrapola a requisição institucional, cuja demanda nos é colocada sem lapidação teórica e ético-política. Só a competência do(a) profissional, pelo conhecimento teórico-político é capaz de decifrar seu significado (CFESS, 2012, p. 30).

As atribuições e competências das(os) profissionais de Serviço Social, sejam aquelas realizadas na saúde ou em outro espaço sócio ocupacional, são orientadas e norteadas por direitos e deveres constantes no Código de Ética Profissional e na Lei de Regulamentação da Profissão, que devem ser observados e respeitados tanto pelas (os) profissionais quanto pelas instituições empregadoras.

Essas atribuições e competências, baseadas na Lei de Regulamentação da Profissão, requisitam do profissional algumas competências gerais que são de fundamental importância à compreensão do contexto sócio histórico em que se situa sua intervenção: a apreensão crítica dos processos sociais de produção e reprodução das relações sociais na perspectiva de totalidade; análise do movimento histórico da sociedade brasileira, apreendendo as particularidades do desenvolvimento do capitalismo no país e as particularidades regionais; a compreensão do significado social da profissão e de seu desenvolvimento sócio histórico; identificação das demandas inseridas na sociedade, visando formular respostas profissionais para o enfrentamento da questão social, considerando as novas articulações entre o público e o privado. (ABEPSS, 1996 apud CFESS, 2010).

São essas competências que permitem ao profissional realizar a análise crítica da realidade, para, a partir daí, estruturar seu trabalho e estabelecer as competências e atribuições específicas necessárias ao enfrentamento das situações e demandas sociais que se apresentam em seu cotidiano.

Na área da saúde, segundo CFESS (2009):

O atendimento direto ao usuário se dá nesses espaços, que na estrutura da rede de serviços brasileira, ganham materialidade a partir dos postos e centros de saúde, policlínicas, institutos, maternidades e hospitais gerais, de emergência e especializados, incluindo os universitários, independente da instância a qual é vinculada seja federal, estadual ou municipal (Ibid., p. 20). 
Desse modo, a atuação profissional deve estar pautada em uma proposta que vise o enfrentamento das expressões da questão social que repercutem, nos diversos níveis de complexidade da saúde, desde a atenção básica até os serviços que se organizam a partir de ações de média e alta densidade tecnológica.

Os/as assistentes sociais atuam na saúde em quatro eixos, são eles: atendimento direto aos usuários; mobilização, participação e controle social; investigação, planejamento e gestão; assessoria, qualificação e formação profissional. Destaca-se que esses eixos não devem ser compreendidos de forma segmentada, mas articulados dentro de uma concepção de totalidade (CFESS, 2010).

\section{ATUAÇÃO DA/O ASSISTENTE SOCIAL NA ONCOLOGIA PEDIÁTRICA}

No Serviço de Oncologia Pediátrica, a atuação do/a assistente social requer intervenções específicas em razão da faixa etária. O primeiro contato do profissional de Serviço Social com o responsável legal pelo paciente infantil é realizado em uma entrevista social para compreensão do quadro sócio familiar, que será útil no processo de tratamento da criança e servirá de subsídio para a reunião com a equipe multiprofissional.

Segundo as assistentes sociais entrevistadas, essa relação interdisciplinar se dá da seguinte maneira:

[...] A gente trabalha também com a Psicologia, que é quando a gente vê que tem um problema mais psicológico a gente faz 0 encaminhamento [...]

(Entrevista Profissional 01).

A gente trabalha muito com a psicologia, terapia ocupacional, com a enfermagem... A gente tem essa interação, porque tem determinados problemas sociais que a gente não detecta e outro profissional pode ajudar. Às vezes a gente vê que têm mães que só faltam surtar e aí a gente conversa com a psicologia pra dar um suporte maior.

(Entrevista Profissional 02).

Na intervenção profissional, também são fornecidas ao acompanhante da criança as orientações necessárias sobre o afastamento do paciente infantil e do acompanhante de suas atividades normais durante o período de internação e tratamento ambulatorial.

Assim, nos centros de tratamento, após a confirmação do diagnóstico do câncer, é proporcionado um atendimento pela equipe multidisciplinar tanto para a criança 
quanto para a família. São oferecidos atendimentos individuais e grupais e o mais comum é que os acompanhantes (pais ou familiares de crianças que recebem tratamento no hospital) sejam beneficiados pelo atendimento psicológico, atuando de modo complementar ao tratamento hospitalar.

A proximidade do/a assistente social do familiar no contexto da assistência hospitalar, constitui um momento privilegiado para que se construa um clima da confiança alicerçado pelo diálogo, no qual haja reciprocidade no falar e no ouvir, fazendo valer o direito do outro se sentir sujeito ativo na comunicação. É com empatia e respeito mediado pela escuta crítica e atenta que permite que se consolide um espaço acolhedor entre o profissional e os pais. O respeito à subjetividade dos sujeitos pautado na valorização do contato humano torna, também, a intervenção do assistente social uma prática do cuidado, como podemos apreender da fala de uma mãe na entrevista:

[...] às vezes as assistentes sociais passam aqui perguntando como é que meu filho tá, passa dando informação, a gente tem reunião em grupo, ela passa algumas informações, o que a gente tem que fazer, sobre os direitos que a gente tem, sobre os benefícios. (Mãe 01)

A ação da escuta qualificada é marcada pela possibilidade de reconhecer as demandas sociais através do conhecimento do outro, o que só ganha sentido na medida em que há uma disponibilidade do profissional para uma abertura à interação dialógica com este outro fato observado, positivamente, durante os questionamentos com as mães, verificada na fala de outra mãe entrevistada:

"É muito bom, a gente poder contar com alguém, saber que tem alguém que se importa, que está disposto a te ajudar. É muito bom saber que a gente não ta na luta sozinha.” (Mãe 02)

Por meio do vínculo criado entre profissional do Serviço Social e usuárioacompanhante, as mães acabam por se sentirem mais à vontade para solicitar a intervenção às assistentes sociais. Para as mães, essa intervenção é vista como uma forma de “ajuda”, não reconhecendo por traz da ação profissional o direito do filho de acesso à saúde. As ações profissionais que se confundem com “ajuda” são recebidas ao demandar uma troca de acompanhante, uma visita extra, uma palavra de conforto, um transporte para voltar para casa, dentre outros. Solicitações essas que são atribuições da instituição e tem no Serviço Social um executor. Essas “atribuições” muitas vezes impedem os usuários de compreender essas ações como parte de seus direitos sociais 
nos remetendo ao passado da profissão, conforme explicitado por uma das mães entrevistadas:

Acho que o papel da assistente social é ajudar. Como se fosse um suporte que o hospital tem que ter. Ela sempre nos ajuda, eu acho que todo hospital tem que ter e também porque ela conversa com a gente. (Mãe 01)

\section{CONSIDERAÇÕES FINAIS}

De acordo com instrumental de pesquisa aplicado e observações participantes dos sujeitos - família - compreende-se que a atuação dos profissionais do Serviço Social no referido Centro Pediátrico do Câncer é percebida pelos pais das crianças que são atendidas na instituição de maneira positiva, porém em uma concepção de "ajuda”, de uma forma amigável e não na perspectiva da garantia de direitos desses usuários preconizada pelo Sistema Único de Saúde.

Nessa concepção, entende-se que tal “ajuda” não se concretiza no processo de acesso à saúde, mas no apoio emocional ao acompanhante, no ato da escuta, no acolhimento ou na orientação.

As famílias tendem a não compreender que todo o trabalho realizado pelas assistentes Sociais é realizado para garantir seus direitos enquanto usuários do serviço público de saúde, e não para ajudá-las de forma "caridosa”, como demonstraram no preenchimento do questionário da pesquisa.

Contudo, conforme assinala Bravo (2006, p. 212), estudos têm apontado que segmentos de profissionais, ao se especializarem na saúde, passam a identificar-se como sanitaristas, pois acabam exercendo outras atividades, distanciando-se da ação do assistente social, que deve passar pela compreensão dos aspectos sociais, econômicos, culturais que interferem no processo saúde-doença e a busca para o enfrentamento destas questões.

Nesse sentido, o profissional do Serviço Social, tanto nesse serviço como em outros serviços na saúde, deve, através de seu conhecimento teórico-metodológico das diferentes expressões da questão social na área da saúde, criar meios para que os usuários possam adquirir consciência de seu direito à saúde e compreendam esta como dever do Estado em provê-la, como também fortalecer o caráter público das ações e serviços de seguridade social. Este profissional deve efetivar a promoção, prevenção, 
proteção, como está posto nos princípios do SUS, podendo ser realizados trabalhos educativos com a comunidade, fortalecendo também as ações e serviços.

Recebido em 04.02.2015 - Reapresentado e Aprovado em 13.10.2015

\section{REFERÊNCIAS}

ACADEMIA NACIONAL DE CUIDADOS PALIATIVOS (2012). O que são

Cuidados Paliativos? [Em linha]. Postado em novembro de 2009. Disponível em: http://www.paliativo.org.br/ancp.php?p=oqueecuidados Acesso em: 17 jun. 2014. ASSOCIAÇÃO DE APOIO À CRIANÇA COM CÂNCER. Câncer Infantil [Em linha]. (s/d). Disponível em: http://www.aacc.org.br/cancer-infantil Acesso em: 24 abr. 2012.

ASSOCIAÇÃo PETER PAN. Associação Peter Pan [Em linha]. Disponível em http://www.app.org.br/ Acesso em: 19 mar. 2012.

BRASIL. Constituição (1988). Constituição da República Federativa do Brasil. Brasília, DF: Senado, 1988.

BRASIL. Lei $\mathbf{n}^{\mathbf{0}} \mathbf{8 . 8 8 0}$, de 19 de setembro de 1990. Dispõe sobre as condições para a promoção, proteção e recuperação da saúde, a organização e o funcionamento dos serviços correspondentes e dá outras providências. Brasília, DF: Ministério da Saúde; Conselho Nacional de Saúde, 1990a. Disponível em: http://conselho.saude.gov.br/legislacao/lei8080.htm. Acesso em: 12 abr. 2014. BRASIL. Estatuto da Criança e do Adolescente. Lei no 8.069, de 13 de julho de 1990. Brasília, DF: Presidência da República, 1990b. Disponível em: http:// www.planalto.gov.br/ccivil_03/leis/18069.htm Acesso em: 29 mai. 2014. BRASIL. Política Nacional de Atenção Oncológica. Publicada em 09 de dezembro de 2010.

Brasília, DF: Ministério da Saúde, 2010. Disponível em: http://www.mpba.mp.br/atuacao/saude/temasemsaude/oncologia/5_Nota_Onco_MS_20 10.pdf Acesso em 15 mai. 2014.

BRASIL. Resolução no 466, de 12 de Dezembro de 2012 [Em linha]. Brasília, DF: Ministério da Saúde; Conselho Nacional de Saúde, 2012. Disponível em: http://conselho.saude.gov.br/resolucoes/2012/Reso466.pdf Acesso em: 30 abr. 2014. 
BRAVO, M. I. S., MATOS, M. C de. Projeto Ético-Político do Serviço Social e sua relação com a Reforma Sanitária: elementos para o debate. In: MOTA, A. E. et al. (Orgs.) Serviço Social e Saúde: formação e trabalho profissional. São Paulo: OPAS, OMS, Ministério da Saúde, 2006, p. 197-217.

CARDOSO, F. T. Câncer infantil: aspectos emocionais e atuação do psicólogo. Rev. SBPH, v. 10, n. 1, Rio de Janeiro, jun. 2007. Disponível em http://pepsic.bvsalud.org/scielo.php?pid=S151608582007000100004\&script=sci_arttext Acesso em: 23 jun. de 2014.

CONSElHo fEDERAL DE SERVIÇO SOCIAL. Parâmetros para a Atuação de Assistentes Sociais na Saúde. Brasília: CFESS, 2009. Disponível em: http://www.cfess.org.br Acesso em: 17 abr. 2014.

CONSElho fEDERAL DE SERVIÇO SOCIAL. Parâmetros para a Atuação de Assistentes Sociais na Saúde. Brasília: CFESS, 2010. Disponível em: eletrônica http://www.cfess.org.br/arquivos/

Parametros_para_a_Atuacao_de_Assistentes_Sociais_na_Saude.pdf Disponível em: 10 mai. 2014.

CONSELHO FEDERAL DE SERVIÇO SOCIAL. Atribuições privativas do/a Assistente Social. $1^{\text {a }}$ ed. amp. Brasília: CFESS, 2012. Disponível em: http://www.cfess.org.br Acesso em: 19 abr. 2014.

CUNHA, A. J. L. et al. Prevenção, Atenção e Controle em Saúde da Criança e do Adolescente. In: PAIM, J. S.; ALMEIDA FILHO, N. Saúde Coletiva: teoria e prática. $1^{\mathrm{a}}$ ed. Rio de Janeiro: MedBook, 2014.

IAMAMOTO, M. V. Conservadorismo e Serviço Social. In: IAMAMOTO, M. V. Renovação e Conservadorismo no Serviço Social: Ensaios Críticos. $9^{\mathrm{a}}$ ed. São Paulo: Cortez Editora, 1992. p. 17-39.

INSTITUTO NACIONAL DE CÂNCER. Particularidades do câncer infantil. (s/d). Disponível em: http://www.inca.gov.br/conteudo_view.asp?id=343 Acesso em: 11 mai. 2013.

INSTITUTO NACIONAL DE CÂNCER. ABC do câncer: abordagens básicas para o controle do câncer / Instituto Nacional de Câncer. Rio de Janeiro: INCA, 2011. 128 p. LIMA, R. A. G. A Enfermagem na assistência à criança com câncer. Ribeirão Preto, SP: AB, 1995.

NORONHA, J. C. LIMA, L. D. MACHADO, C.V. O Sistema Único de Saúde. In: GIOVANELLA L., ESCOREL S., LOBATO L.V.C., NORONHA J.C., CARVALHO 
A.I, (Orgs). Políticas e Sistema de Saúde no Brasil. Rio de Janeiro: Editora Fiocruz; 2008.

NÚCLEO DE APOIO À CRIANÇA COM CÂNCER. Câncer Infantil/Sintomas [Em linha]. (s/d). Disponível em: http://www.nacc.org.br/cancer-infantil/o-que-e-cancer/ Acesso em: 15 jul. 2012.

VALLE, E. R. M. Vivências da família da criança com câncer. In: CARVALHO, M. M. M. J. (Org.). Introdução à Psico-oncologia. São Paulo: Psy II, 1994. 
